\title{
Development and control of a shifting performance test bench equipped with AC servo drive shifting manipulator
}

\author{
Yong Luo ${ }^{1, \mathrm{a}}$, Yu-Feng Cao ${ }^{1, \mathrm{~b},+}$, Xue Zhao ${ }^{1, \mathrm{c}}$ and Ling-Yu Feng ${ }^{1, \mathrm{~d}}$ \\ ${ }^{I}$ College of Vehicle Engineering, Chongqing University of Technology, Chongqing, \\ 400054 P.R. China. \\ ${ }^{a}$ cquluo@126.com, b449058606@qq.com, c876421149@qq.com, \\ ${ }^{d}$ cqfenglingyu@163.com \\ ${ }^{+}$Corresponding author
}

\begin{abstract}
Shifting performance of Manual Transmission is very important for users and manufactures of Manual Transmission. Traditionally, shifting performances were tested on test bench equipped with pneumatic shift manipulator, which was low efficiency, low control accuracy and noisy. In order to improve test efficiency and accuracy, a shifting performance test bench equipped with AC servo drive was built. Two AC servo drive motors were used as the actuating device of shifting manipulator. Control methodology of these two AC servo drive motors was established to meet the needs of shifting performance testing with high efficiency and accuracy. Test results indicated that the shifting process ran smoothly and shifting performance of Manual Transmission can be tested with high efficiency and high accuracy on this test bench.
\end{abstract}

Keywords: Shifting performance test bench, Shifting manipulator, AC Servo Drive.

\section{Introduction}

Shifting performances of manual transmission in different speed have significant influence on driving performance. Traditionally, shifting performances were tested on test bench equipped with pneumatic shift manipulator, which was low efficiency, low control accuracy and noisy[1]. AC servo drive motors were wildly used nowadays, they can be controlled more accurately than pneumatic cylinder. AC servo drive motors also have much quicker control response than pneumatic cylinder which means higher test efficiency[2]. In consider with all these advantages, a shifting performance test bench equipped with $\mathrm{AC}$ servo drive shifting manipulator was built In order to improve test efficiency and accuracy. Measurement and control system of the AC servo drive shifting manipulator was designed based on PLC controller. Control methodology of these two AC servo drive motors was established to meet the needs of shifting performance testing with high efficiency and accuracy. The system was built and tested, test results indicated that the shifting process ran smoothly and shifting 
performance of Manual Transmission can be tested with high efficiency and high accuracy on this test bench.

\section{Working Principle of the Test Bench}

Structure of shifting performance test bench was shown in Figure1. A Frequency conversion motor was used as driving motor to provide the test bench with power so the whole test bench can rotate. An inertial flywheel was connect with the drive motor by a twin universal joint coupling, which was use to simulate driving inertia of vehicle. Other side of inertial flywheel was connected with the output side of testing gearbox, a torque and speed sensor was set between inertial flywheel and output side of gearbox to measure torque and rotate speed. Input side of the gearbox was connected with nothing, because engine and gearbox were disengaged during shifting. Shifting manipulator using AC servo drive motors was used to change gears of the gearbox[3-4]. Structure of shifting manipulator using AC servo drive motors was shown in fig. 2. There are two AC servo drive motors in the shifting manipulator, one is used as gear selector, and the other is used shift gear. With these settings, shifting process of test gearbox can be simulated just as in real cars, and shifting performance of the test gearbox can be tested in this test bench.

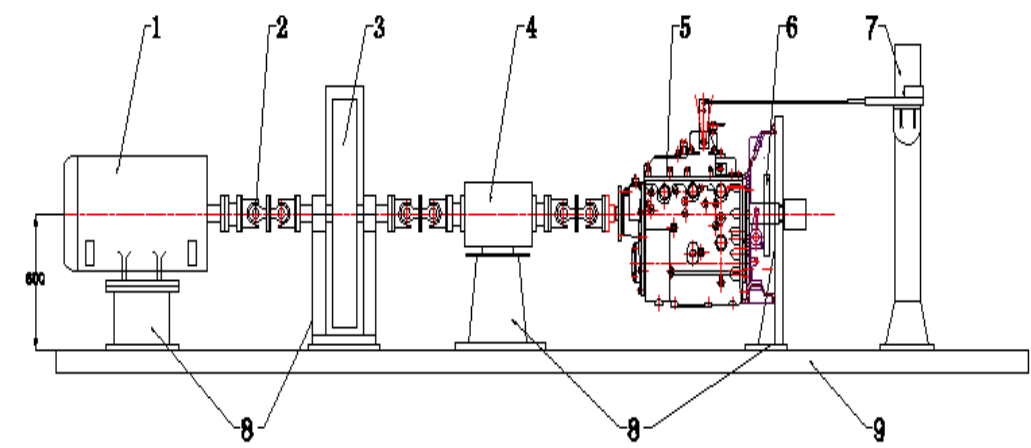

1 Driving motor; 2 Twin-universal joint coupling; 3 Inertial flywheel; 4 Torque and speed sensor; 5 Test gearbox; 6 Inertial plate; 7 Shifting manipulator; 8 Mounting bracket; 9 Cast iron platform Figure 1. Structure of shifting performance test bench 
Figure 2. Shifting manipulator using AC servo drive motors

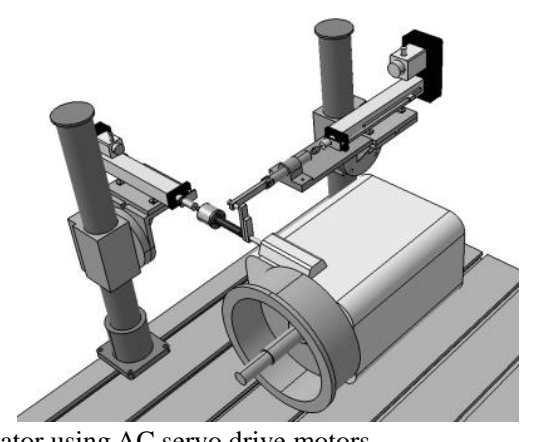

\section{Measurement and Control System of AC Servo Drive Shifting Manipulator}

The measurement and control system of shifting manipulator was shown in figure 3. The system was built up with three parts, including control system, actuating system and data collection system. The control system contains an Industrial Personal Computer (IPC) and a PLC, the IPC was connected with PLC by Ethernet cables. IPC used to store data collected by data collecting system, and sending control command to PLC. PLC send control signals to actuator of servo motors, which control servo motors to rotate. Actuating system is consisting of two servo motors and their actuators, and two screw rods. Actuators receive control signals from PLC and controls serve motors to rotate, screw rods change the rotating into linear motion, so as to shift the gearbox. Data collection system collect displacement of the two screw rods and push force of the screw rods, these data were transmitted to the Control system.

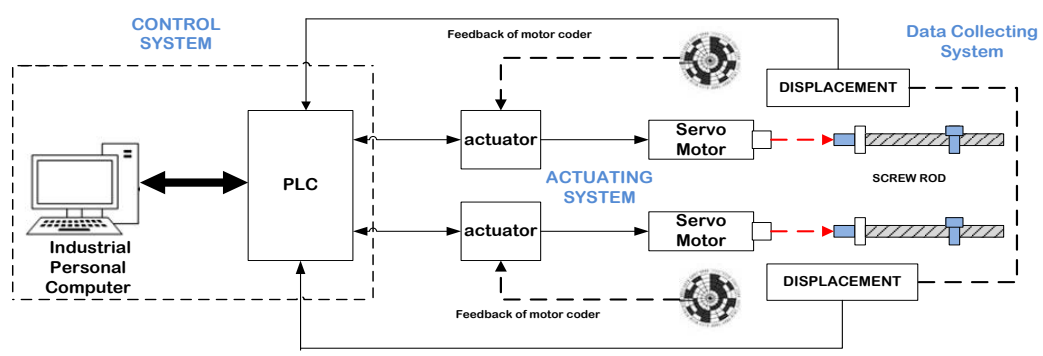

Figure 3. Measurement and control system for AC servo drive shifting manipulator

\section{Measurement and Control System of the Test Bench}

Measurement and control system of the test bench was shown in figure 4. An IPC was use to control the whole system and collecting data. A data collection card was used to collect rotation speed of the servo motors and force in the 
screw rods, the data collection card was connected to the IPC using PCI interface. Torque and speed sensor measure torque and rotate speed in the output side of test gearbox, the signals were transmitted to a signal modulate instrument to change in to digital signal. The signal modulate instrument was connected to IPC by RS232 data bus. PLC send control signals tor actuator of servo motors and the driving motor using control command transmitted from IPC. PLC was connected with IPC by crosslink Ethernet cable, it receives commands from IPC and feedback working conditions of servo motors and driving motors to IPC.

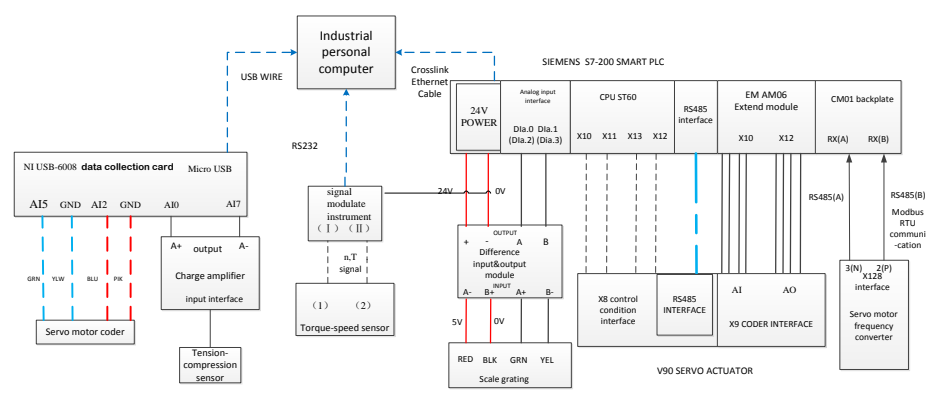

Figure 4. Measurement and control system of the test bench

\section{Develop of Control Software}

The system uses PLC to control servo motors and driving motor, and use IPC to system measurement and control. Measurement and control software in IPC was developed using LabVIEW. LabVIEW don't support Siemens communication protocol, OPC(OLE for Process Control) was used to transmit data between IPC and PLC.

\subsection{Control software in PLC}

PLC control software contain six parts, including manual operation, calibration, normal test, single step operation, back to neutral gear, driving motor control. The control logic of the whole main program is shown in figure 5. 


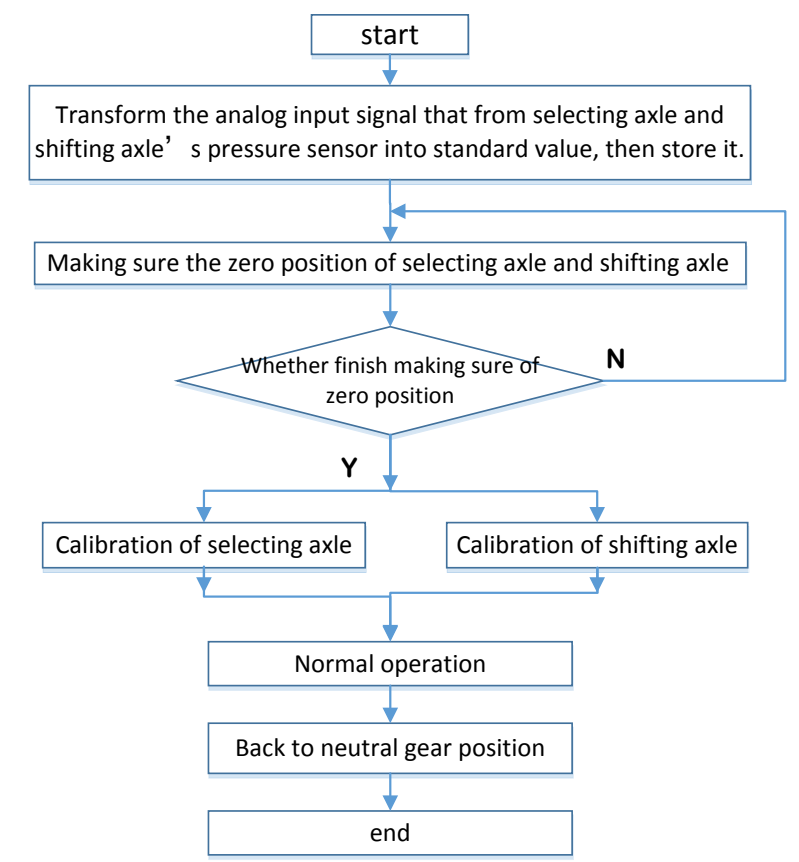

Figure 5.Control logic of PLC

\subsection{Measurement and control software based on LabVIEW in IPC}

There are five parts in measurement and control program, including test setting, gear calibration, test control (control of shifting manipulator, control of driving motor), state monitoring, and data processing. The control logic of measurement and control program is shown in figure 6 .

Test setting module include test process setting and test parameters setting, test process setting include test date setting, selection of working condition, shift times and etc. Test parameters setting include up limit of gear select and shift force, velocity limits of gear select and shift screw rod. Under calibration mode, we can move gear select and shift servo motor by hand operation, we operation several buttons to move the servo motors to change the shift lever to different gear position. Coordinate of each gear was remembered by the software, these positions was latter used in test control mode to decide move direction and distance of each screw rod under different shift conditions. Under test control module, the program controls the system automatically to procedure the test process. State monitoring module was used to 
collect and display different parameters of the system, so the operator knows the working condition of the system. Data processing module used to handle the tested data, so we can get more information about shifting performance of the test gearbox.

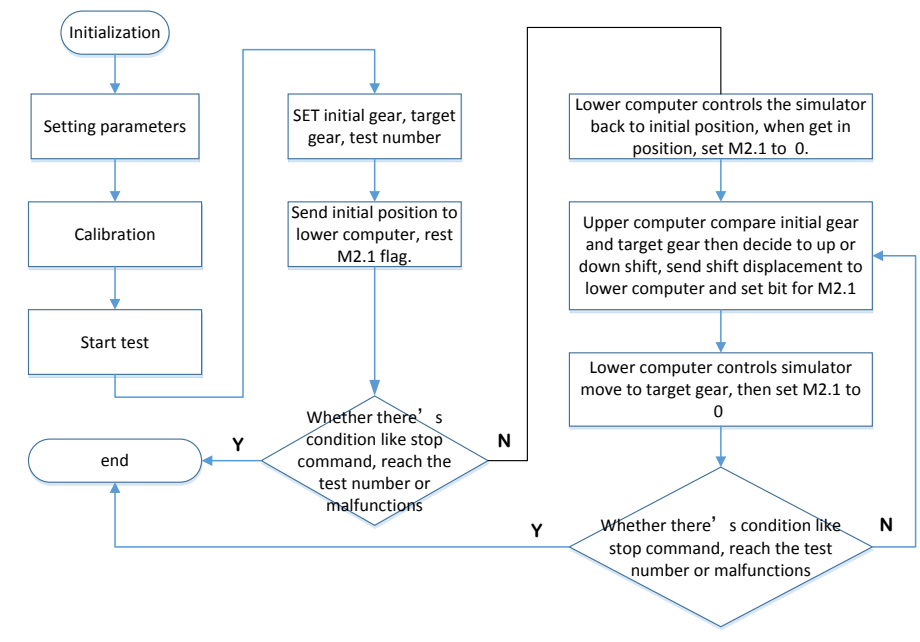

Figure 6. Control logic of upper computer

\section{Analysis for Test Result}

The test bench was built and tested, Pictures of the shifting performance test bench and measurement and control program are shown in figure 7. One of the tested curves of shifting force is shown in figure 8 .

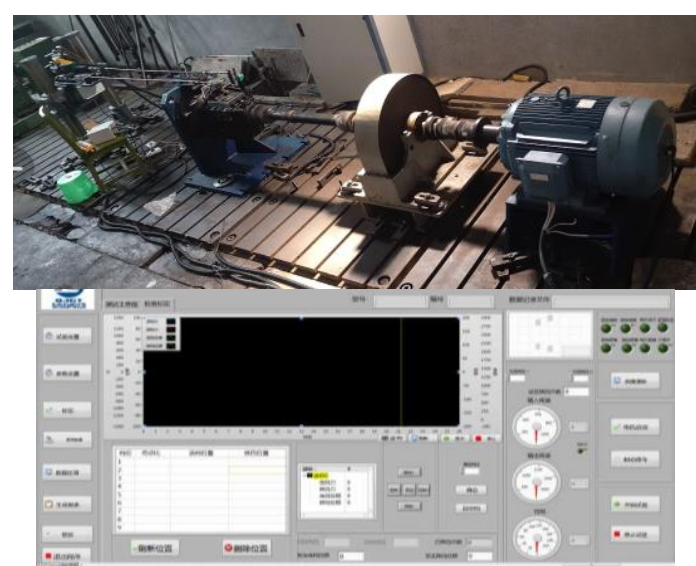

Figure 7. Pictures of the test bench and measurement and control program 


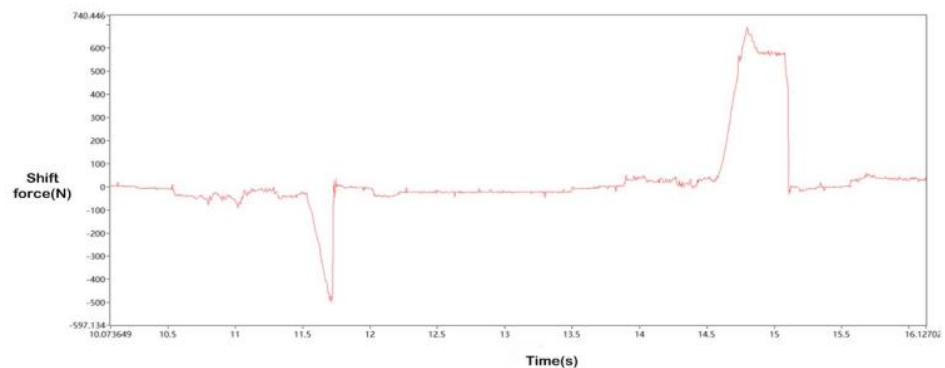

Figure 8. Curve of shifting force

Figure 8 shows the tested curve of shifting force in a test cycle in which gear changes between gear 2 and gear 3 . According to Figure 8 , direction of the force is opposite when gear 2 changes to gear 3 and gear 3 changes to gear 2 . From 11.5 s to $12 \mathrm{~s}$, the shifting process is gear 2 changes to gear 3 , we can see from the shifting force that the synchronization process is not obvious. During the time of $14 \mathrm{~s}$ to $16 \mathrm{~s}$, the gear changes from 3 to 2 , the force changes dramatically, and the synchronization process is very clear.

\section{Summary}

A shifting performance test bench equipped with AC servo drive shifting manipulator was designed and built, a PLC is used in the system to control the two servo drive motor and driving motor, a IPC is used in the system to run measurement and control program based on Labview. Two AC servo drive motors were used as the actuating device of shifting manipulator. Control methodology of these two AC servo drive motors was established to meet the needs of shifting performance testing with high efficiency and accuracy. Test results indicated that the shifting process ran smoothly and shifting performance of Manual Transmission can be tested with high efficiency and high accuracy on this test bench.

\section{Acknowledgement}

This project is supported by the National Natural Science Foundation of China (Grant No. 51305475); This research is funded by Chongqing Research Program of Basic Research and Frontier Technology (Grant No. cstc2013jcyjA60004); This research is funded by Scientific and Technological Research Program of Chongqing Municipal Education Commission (Grant No. KJ1500927).

\section{References}

[1] Ling Ling, Wang Fei, Xiao Yu-Bo. Experimental investigation of the 
cryogenic properties of automatic mechanical transmissions [J]. Proceedings of the Institution of Mechanical Engineers, Part D: Journal of Automobile Engineering. 2016, (230):1173-1181.

[2] Chyuan-Yow Tseng, Chih-Hsien Yu. Advanced shifting control of synchronizer mechanisms for clutchless automatic manual transmission in an electric vehicle [J]. Mechanism and Machine Theory. 2015, (84):37-56.

[3] Ide Takanori, Kitajima Hiroyuki, Otomori Masaki, Leiva Juan Pablo, et.al. Structural optimization methods of nonlinear static analysis with contact and its application to design lightweight gear box of automatic transmission of vehicles [J]. Structural and Multidisciplinary Optimization. 2016, (53):1383-1394.

[4] Zhao Xinxin, Yang Jue, Zhang Jing, Zhang Wenming. Optimizing gear shifting quality with backstepping control for automatic transmission [J]. Transactions of the Chinese Society of Agricultural Engineering. 2016, (32):27-33 